\title{
Ferrajoli, Luigi, Principia iuris. Teoria del diritto $e$ della democrazia, Laterza, Bari-Roma, 2007, 3 vols. (Vol. I: 1.021 pp.; vol. II: 713 pp.; vol. III: 1.002 pp).
}

El profesor Ferrajoli es conocido en nuestro medio por su extraordinaria obra jurídica, la que ha influido de manera decisiva sobre la teoría del derecho en general y sobre la dogmática jurídica, y en esta notándose su influjo especialmente en la dogmática de los derechos fundamentales y del proceso penal.

Se ha difundido con especial fuerza, entre nosotros, Derecho y razón. Teoría del garantismo penal (1989), obra que se ha convertido en referencia obligada en el estudio de las garantías penales y procesales penales, así como otras publicaciones de gran relieve en la teoría general del derecho y de los derechos fundamentales, como Derecho y garantías. La ley del más débil (1999); Sobre los derechos fundamentales y sus garantías (2006), y también Epistemología y garantismo (2006), entre otras.

Ahora publica Principia iuris. Esta se insinúa como una suerte de corolario o gran propuesta teórica integral del autor, en donde asume y propone de forma unitaria y global lo que antes había desarrollado de manera parcelada. Se trata de una obra monumental por su extensión y pretensión omni-comprensiva y, al mismo tiempo, por su rigor teórico.

\section{Primera eXPlicación PREVIa: CONTENIDO DE LA TEORÍa Y LA METATEORÍA}

La teoría abarca o comprende cuatro partes: la deóntica, que contiene la parte destinada al estudio de las formas y las estructuras generales del derecho y las relaciones que se producen dentro de ella entre sus distintos términos y conceptos fundamentales. El estudio del derecho positivo, parte en la cual se aborda el análisis de este en sus aspectos y elementos esenciales. Esta segunda parte está íntimamente vinculada a la tercera referida al estudio del Estado de derecho. Para la comprensión tanto de la segunda como de la tercera resulta fundamental el análisis y comprensión de la teoría de los derechos y las garantías y sus términos correlativos de laguna y antinomia. He aquí a mi juicio el núcleo de aportaciones más importantes de Ferrajoli en lo que hace a su teoría del derecho, y a partir de ese núcleo se comprende el carácter nomodinámico y multinivel del ordenamiento positivo moderno y las consecuencias extraordinariamente importantes que deriva de estas características el teórico italiano.

En fin, la cuarta parte está destinada al estudio de la democracia cons- 
titucional, a la que dedica todo el volumen II. Se trata de una teoría de la democracia constitucional que constituye una interpretación normativa y semántica de la teoría del derecho, coherente con ella. Se defiende, en lo fundamental, la necesidad de una concepción no puramente formal o procedimental de democracia, sino además sustancial, como un imperativo de la democracia constitucional avanzada. En segundo lugar, para el jurista chileno resultará interesante constatar la sólida y extensa exposición que se hace de la democracia social como forma que supera a la democracia liberal, y que por ello constituye una ampliación y perfeccionamiento del Estado constitucional de derecho.

Pero antes de referirse a las cuestiones fundamentales de la teoría cabe aclarar ciertos aspectos esenciales de la denominada metateoría del derecho, es decir, de la teoría de la teoría o, mejor, de las formulaciones sobre lo que la teoría es, cómo se justifica y cómo se construye. Desde estos puntos de vista la metateoría comprende la semántica, la pragmática y la sintáctica de la teoría del derecho.

La semántica. Esta se refiere a la determinación del objeto de indagación del discurso teórico, el significado del mismo respecto a una realidad analizada, indagada por la teoría.

Para ese fin ha de distinguirse en primer lugar la extensión de la teoría del derecho, que se identifica con los ámbitos de experiencia a los cuales ella es aplicable. La teoría de Ferrajoli es una teoría del derecho positivo moderno existente y desarrollado dentro de formas de democracias constitucionales avanzadas. Es ese el marco de referencia empírica directo e inmediato y a él atiende o apunta su potencialidad explicativa.

En segundo lugar, la aproximación semántica ha de determinar la denominada intensidad (intensión) de la teoría. Ella se concibe como su "concreto contenido informativo, es decir, su referencia semántica a los fenómenos empíricos sobre cuya base es interpretable. Será por lo tanto posible configurar la teoría del derecho, dentro de un modelo integrado de ciencia jurídica, como un sistema de conceptos y de asertos teóricos interpretables, en la línea de los fenómenos esenciales como referencias empíricas, por dos tipos de discursos - la dogmática jurídica y la sociología del derecho- entre ellos distintos y, sin embargo, conexos como las caras de la misma medalla. Será además posible asociar a gran parte de los conceptos y de las construcciones teóricas también una interpretación axiológica: aquella expresada por la valoración y la proyección del derecho utilizada en la filosofía de la justicia." (Vol. I, pp. 8-9).

En esta línea se puede decir que las dos referencias empíricas de la teoría vienen dadas por las normas y los hechos. La comprensión de la referencia normativa (las normas) de la teoría integra a ella el punto de vista y el análisis de la dogmática jurídica. Por otra parte, los hechos desde una perspectiva teórica son una segunda referencia empírica asumida y de esa manera queda recogida la visión de análisis de la sociología jurídica. Para 
el autor ambas aproximaciones deben estar integradas en la teoría del derecho si se quiere hacer un análisis del objeto completo. La aproximación de la dogmática al ser eminentemente normativista se ocupa de la norma: "del análisis del 'lenguaje legal', de la interpretación jurídica de los datos empíricos que son los enunciados normativos de que está compuesto el discurso del legislador. Por el contrario, el universo del discurso de la sociología (y de las teorías del derecho de enfoque realístico), consistiendo en un universo en larga medida extra-lingüístico, requiere prevalentemente como métodos de investigación de los instrumentos de investigación sociológica, es decir, de la relevación, de la descripción o de la explicación de los hechos y los comportamientos jurídicos." (Vol. I, pp. 9-10).

Ambas aproximaciones deben ser recogidas en una teoría del derecho que se refiere a un sistema jurídico positivo multinivel, ya que siempre en ellos "todos los fenómenos normativos pueden ser considerados, simultáneamente, como hechos (o actos) y como normas. Precisamente, las normas pertenecientes a los niveles normativos inferiores vienen en ellos considerados además como actos (puesto que tales divergentes) respecto a las normas de nivel superior que regulan la producción. Inversamente, los actos normativos pertenecientes a los niveles normativos superiores vienen relevados además como normas (puesto que tales virtualmente divergentes) respecto a los actos normativos inferiores de los cuales regulan la producción." (Vol. I, p. 12).

Con ello el profesor italiano pone de relieve que la teoría necesariamente ha de recoger la doble implicación inevitable que se da entre norma-acto y hecho en un ordenamiento complejo multinivel, lo cual además resulta necesario para hacer posible el carácter crítico y proyectual en el tratamiento sistemático de las imperfecciones normativas del derecho positivo expresadas en las antinomias y lagunas.

Con ello se evitan las falacias reduccionistas normativista y realista. La normativista que describe y se ocupa "solo del derecho que (normativamente) debe ser, como en la aproximación normativista que ignora el derecho que es, o sea, la práctica efectiva." (Vol. I, p. 14). La falacia reduccionista realista por la cual solo se atiende al "derecho que (efectivamente) es, como en la aproximación realista que ignora el derecho que debe ser, o sea, su modelo normativo cual ha sido diseñado por la constitución." (Vol. I, p. 14). Y concluye el autor: "Resultan dos imágenes opuestas, pero entre sí parciales y distorsionadas del derecho, dado que una descuida los márgenes de inefectividad de la norma de nivel superior y la otra aquellos de ilegitimidad de las normas de nivel inferior." (Vol. I, p. 14).

Como ya hemos dicho la teoría debe asumir la necesidad de acoger ambas aproximaciones si quiere ser una teoría realmente explicativa del derecho positivo moderno y multinivel y quiere entonces comprender el fenómeno de la validez normativa y de la existencia, junto al de efectividad e inefectividad, fenómenos jurídicos inevitables y que hacen a la 
esencia del objeto de estudio de la teoría. Así "La metateoría semántica impone en suma a la teoría del derecho la necesidad de tematizar esta ambivalencia de los fenómenos normativos: de distinguir la existencia y vigor tanto de su validez como de su efectividad y por esto de leer como validez o invalidez de las normas de nivel inferior la efectividad o inefectividad de las normas de nivel superior, y las unas y las otras como conformidad y deformidad (cuanto a la forma) o como coherencia e incoherencia (cuanto al significado) de las primeras respecto a las segundas. La teoría así construida será por lo tanto al mismo tiempo normativista y realista, dado que tales fenómenos serán capaces de explicar sea la naturaleza de normas como aquellas de hechos, no ya como universos distintos sino como las dos caras del mismo universo, cuyas divergencias reflejan precisamente la estructura deóntica o normativa," (Vol. I, p. 15).

De esto depende que se pueda cumplir la principal función de la ciencia jurídica (en un modelo integrado) como es la identificación de antinomias y lagunas, es decir, de identificar el "derecho ilegítimo" en cuanto inválido o lagunoso, en cuanto imperfecciones del derecho, y de promover las formas de superación de ellas.

La pragmática. La dimensión pragmática de la teoría quiere decir primeramente que ella no es avaluativa, ideológicamente neutral, sino al contrario ha de reconocer o explicitar los aspectos que suponen consideraciones valorativas u opciones ideológicas realizadas por el teórico. Un primer aspecto de la teoría que revela la dimensión pragmática de la teoría es aquel que hace referencia a los conceptos y postulados utilizados por la teoría y que "son el fruto de definiciones 'estipulativas' o 'asunciones', es decir, de decisiones justificadas no ya por los usos linguísticos del legislador sino por la estrategia explicativa puesta en actuación por el mismo teórico." (Vol. I, p. 22). Este primer aspecto de la dimensión pragmática en la teoría axiomática de Ferrajoli se declara de forma explícita y completa. Cada definición estipulativa, cada asunción están expresamente reconocidas y determinadas.

Una segunda dimensión pragmática viene dada por la consideración del derecho positivo nomodinámico y multinivel el cual se halla caracterizado por el "deber ser" normativo y el "ser" efectivo, y cuyo reflejo o correlativo a nivel teórico se expresa en los denominados principia iuris tantum y principia iuris et in iure. Los principia iuris tantum como principios de la lógica aplicados al derecho -son el principio de no contradicción y de completitud- tienen a nivel teórico una función descriptiva puesto que describen la relación teórico-ideal del derecho positivo, pero junto con ello tienen una función normativa en el derecho positivo en cuanto proyectan o expresan la necesidad en o dentro del derecho positivo multinivel de que las normas subordinadas a normas supraordinadas se somentan a esta. De ese modo los principia iuris tantum tienen una función normativa con relación a los principia iuris et in iure consagrados en 
normas de derecho positivo supraordinadas, las cuales poseen efectividad, por decirlo de una manera gráfica, demandada a nivel teórico por los principia iuris tantum. Y precisamente por ello esta dimensión pragmática de la teoría puede recoger o comprender los fenómenos que suponen imperfecciones del derecho o la existencia del "derecho ilegítimo" (que de otra forma es manifestación del binomio: norma-hechos), y, por su medio, se logra tematizar y sistematizar el análisis de las denominadas antinomias y lagunas, y además identificar los medios (garantías) para reducir las divergencias normativas en el derecho positivo. Es evidente el carácter pragmático en ello.

La sintáctica. En esta parte de la (meta) teoría se pretende formular y explicitar la manera en que se construye el discurso teórico. Con ese fin aclara el tipo de lenguaje del que se servirá, lo cual resulta además fundamental para una teoría como la de Ferrajoli, definida por él mismo como teoría formalizada. Se explica que la ciencia jurídica requiere del uso de dos lenguajes -el observativo y el teórico-, los cuales utilizará en discursos de niveles distintos.

El lenguaje observativo es el utilizado por las ciencias empíricas. A través de él se describen realidades, procesos o relaciones que entre aquellas se puedan verificar. La ciencia jurídica utiliza este lenguaje en el nivel de la dogmática jurídica, en donde el objeto de análisis resulta la norma jurídica positiva respecto de la cual la dogmática, a través de un lenguaje observativo, realiza aserciones de tipo existencial sobre el vigor, sobre la validez, o sobre sus significados. Es evidente, sostiene el autor, que el lenguaje observativo de la dogmática es altamente técnico por la misma naturaleza de su objeto que es en sí mismo un universo lingüístico analizado a través de redefiniciones de los conceptos y la interpretación de los enunciados expresados por el legislador. Y por eso concluye: "El lenguaje de la dogmática no es por lo tanto el genérico y elemental lenguaje común, sino un lenguaje altamente técnico, cuyos términos tienen sus reglas de uso en el discurso legislativo y cuyos asertos valen para explicitar y analizar, de este mismo discurso, la lógica interna." (Vol. I, p. 46).

Por el contrario la teoría del derecho (dentro de la ciencia jurídica en un modelo integrado) que pertenece a un nivel de discurso distinto del de la dogmática, ya no observativo de normas, sino teorizante y por lo tanto destinado a la elaboración de definiciones estipulativas o convencionales creadas, establecidas por el teórico y "libremente asumidas con vista a la finalidad explicativa perseguida por la teoría", ha de utilizar un lenguaje artificial y convencional construido enteramente por el teórico: "el lenguaje teórico de la teoría del derecho, no teniendo referencia directa al discurso normativo, es un lenguaje artificial y convencional, es decir, enteramente construido por el teórico y por lo tanto formalizable según una situación rigurosamente preconvenida." (Vol. I, pp. 47-48). 


\section{SEGUNDA EXPLICACIÓN PREVIA: TEORÍA AXIOMÁTICA DEL DERECHO}

En la obra se propone una teoría del derecho axiomática, es decir, que se vale de un lenguaje axiomatizado, en donde el autor construye su formulación sobre la base de que cada elemento conceptual se explicite o defina: de allí la explícita determinación de los 16 postulados originarios y los 274 conceptos definidos, sobre los cuales estructura su teoría y cada uno de los teoremas que la componen, que llegan a 1.679, todos sometidos a control de demostración lógica. El método axiomatizado que propone Ferrajoli ya había sido parcialmente expuesto en Derecho y razón, pero esta vez se explicita y desarrolla cabalmente. El método asegura precisión en lo que el autor denomina la sintaxis del derecho: ninguna premisa primitiva -postulados originarios y definiciones- queda sin expresa declaración o determinación; ningún teorema se supone demostrado de forma intuitiva, sino precisa y claramente sometido a control de verificación lógica. Con ello construye una teoría del derecho con pretensiones de integridad y coherencia formalmente verificables. De esa manera se logra disminuir la imprecisión y la equivocidad a su mínima expresión, y facilita y promueve la detección de los posibles errores y lagunas de la teoría. Esto último resulta un homenaje a la máxima seriedad y honestidad científica. Dice el autor que en el método axiomatizado es evidente que cada modificación de una tesis primitiva, si repercute sobre todas las tesis sobre las cuales opera directa o indirectamente, y sirve de premisa, comportará la carga de modificar toda la cadena de las tesis de ella directa o indirectamente derivadas. Pero son precisamente estas modificaciones, además de la introducción de nuevos postulados o de nuevas definiciones, que acrecentando la complejidad y la capacidad explicativa de la teoría, determina la precisión, la corrección, las integraciones y el desarrollo. El empleo del método axiomático, en breve, hace posible la ampliación y el progreso científico de la teoría (Vol, I, pp. 21 y ss., introducción, y Vol. III, p. 5).

Es evidente que la teoría del derecho se expresa en un lenguaje artificial o convencional creado por el teórico. Por eso la necesidad de que cada concepto esté definido dentro de un sistema teórico, eso permitirá no solo verificar la precisión lógica del mismo, sino también su capacidad explicativa.

Realizada las explicaciones previas atendamos en seguida a los aspectos fundamentales de la teoría del derecho propuesta en Principia iuris.

\section{LA OBRA PARTE DE LA BASE DE UN DESARROLlO DE CAMBIOS CONSE- CUTIVOS DE PARADIGMAS EN LA CONCEPCIÓN DEL ORDENAMIENTO JURÍDICO HASTA NUESTROS DÍAS}

En lo medular sostiene que el primer modelo o paradigma suponía que el ordenamiento se estructuraba sobre la base de una norma de reco- 
nocimiento que inicialmente, en el derecho premoderno, venía dada por la sustancia de la norma, es decir, por la convicción de que ella era justa, o se correspondía con un concepto de justicia, lo cual era exactamente declarado positiva o negativamente en la sentencia del juez. Derecho y justicia tendían a confundirse y detrás del concepto de justicia se hallaban predeterminaciones sustanciales de carácter moral. Para Ferrajoli este primer modelo de justicia desaparece con la irrupción del principio de legalidad hacia fines del siglo XVIII. Desde ese mismo momento se modifica la forma de reconocimiento del derecho: ya no es la sustancia de la norma sino la forma de producir el derecho; se reconoce el derecho en la medida que se ha producido de una manera. En ello se apoya el sometimiento del juez a la ley y la igualdad ante la ley. En el momento mismo en que se basa la legitimidad y reconocimiento en la forma de producirlo, se promueve un abandono de criterios sustantivos o materiales de justicia de la norma. Se produce entonces una separación del derecho y la moral. La legalidad, que parece y tiene una fuerte connotación autoritaria, adquiere modernamente una fuerte connotación de garantía. Así en el derecho penal la legalidad es una gran garantía para la certeza jurídica. Por eso el principio de estricta legalidad supone que la legalidad posea una intencionalidad y de esa manera hace posible la determinación de su extensión. Pero para eso la ley debe describir con términos precisos que posean poder de verdadera connotación de realidades empíricas inmediatas, de manera que excluyan el uso de términos ambiguos o abiertos sin referencia inmediata a la realidad.

El segundo cambio de paradigma ha consistido en el surgimiento del constitucionalismo, que ha venido a sostener que la constitución opera como una norma supraordinada a la ley y que impone un cierto contenido, afirmación fundamental pues importa en la concepción de Ferrajoli la superación del paleopositivismo por un positivismo de garantía. La norma constitucional impone, en efecto, determinados contenidos como la igualdad, las libertades o los derechos sociales, contenidos sustanciales impuestos a la norma subordinada. La norma inferior, ciertamente podría infringir esos contenidos, pero ella debe -por esa divergencia- ser anulada, expulsada del ordenamiento justamente por no someterse a la norma supraordinada. De aquí se desprende el carácter complejo de los ordenamientos constitucionales como sistemas jurídicos nomodinámicos y con más de un nivel normativo (multinivel). El nivel inferior, el legal, se somete al nivel superior. Ello importa la existencia de características bien precisas del ordenamiento jurídico.

Desde esta perspectiva es fundamental anotar que para el autor el consenso mayoritario no garantiza la validez normativa. Ninguna mayoría puede tocar la esfera de lo indecidible delimitada por los derechos y las garantías del constitucionalismo, lo cual supone que el ordenamiento sí posee una referencia sustantiva, lo mismo que la democracia. Por eso la naturaleza de la democracia no está sin más en la soberanía, pues hay 
límites que excluyen materias o cuestiones del poder soberano, como los derechos fundamentales. Lo anterior se corresponde con el concepto de democracia sustancial, que es aquella referida a los contenidos constitucionales vinculantes, los derechos fundamentales, y que vincula al Estado de derecho constitucional con dicha forma determinada de democracia, la sustancial, es decir, aquella respetuosa de los derechos fundamentales que corresponden a todos, y no a una parte o a la mayoría (Vol. I, pp. 486 y ss., $§ 9.2$. Vol. II, pp. 35 y ss., $§ 13.7$ y 13.8 , y pp. 303 y ss., $§ 15.1$ ).

\section{PRINCIPIA IURIS TANTUM Y PRINCIPIA IURIS ET IN IURE. EL DERE- CHO POSITIVO, ENTRE EL DEBER SER NORMATIVO Y EL SER EFECTIVO (LA PRAGMÁTICA)}

Dijimos al inicio de este comentario, como breve explicación sobre una de las dimensiones de la pragmática de la teoría, que la consideración en ella de los denominados principia iuris tantum y principia iuris et in iure constituye la forma teórica por la cual, podríamos decir, se recoge el carácter normativo y al mismo tiempo empírico-efectivo del derecho, y la necesidad de describir y también de proyectar teóricamente el fenómeno indicado.

La concepción teórica del derecho de Ferrajoli importa una determinada concepción de la ciencia jurídica y del sistema jurídico positivo. Por eso afirma que la idea misma de sistema jurídico como sistema unitario postula una ciencia jurídica no puramente recognitiva, sino a su vez normativa, con fuerza en dos clases de principios distinguidos: los principia iuris tantum expresados por la lógica y externos al derecho, y los principia iuris et in iure, internos o propios del derecho, reformulados por la dogmática y reelaborados por la teoría, lo cual es tanto o más real en el paradigma que, por un lado, está marcado por desniveles normativos y, por lo tanto, por las virtuales presencias de antinomias y de lagunas, y que, por otro lado, ha positivado las elecciones a las que el derecho debe uniformarse y que hacen por esto parte del objeto de la indagación de la ciencia jurídica. Por estó afirma que la dogmática y la teoría del derecho no pueden limitarse a decir aquello que el derecho es, ya que no pueden no decir también aquello que el derecho debe ser e igualmente hace parte -a un nivel normativo diverso- del derecho que es, además de aquello que el derecho no debe ser y que sin embargo es. Precisamente mientras la teoría enuncia las condiciones formales y sustanciales de validez de las normas jurídicas, la dogmática identifica los límites y los vínculos de contenido concretamente impuestos, a la validez de las normas de un determinado ordenamiento, por las diversas clases de principios jurídicos a ellas supraordinados (Vol. I, pp. 30-31).

Según el autor su teoría se propone como una teoría garantista con la inevitable dimensión pragmática de la misma. El paradigma constitucio- 
nal es un paradigma formal que puede tener, dice, cualquier contenido, ya que responde al paradigma iuspositivista. Es en este carácter formal en el que reside su valor teórico-jurídico cual modelo normativo vinculante más allá de los principios constitucionalizados, conjuntamente a su valor teórico-político, cual fundamento de la separación laica entre derecho y moral y, en conjunto, con la autonomía y el primado del punto de vista político externo al derecho vigente, crítico y proyectual en su contraposición, incluidas las mismas constituciones. Pero, añade, que no obstante lo dicho la referencia empírica de su teoría es la experiencia histórica del constitucionalismo democrático y su capacidad expansiva. Pertenece por eso a la teoría de la democracia la identificación de las diversas garantías con capacidad de reducir la desviación entre el deber ser y el ser efectivo del derecho positivo (Vol. I, p. 31). La teoría de Ferrajoli es positivista en cuanto niega la preexistencia de contenidos necesarios en las construcciones jurídicas teóricas, pero cada construcción teórica -y por eso la suya también- ha de poseer una referencia empírica y valórica que de manera explícita o implícita le anime. En el caso de Principia iuris ella viene formulada como una teoría jurídica sobre el derecho y la democracia en un modelo de constitucionalismo democrático avanzado.

Por lo anterior es que añade de manera coherente que el carácter puramente formal y estructural de la teoría del derecho en el sentido propio no nos dice nada sobre los contenidos del derecho positivo ni comprenda un compromiso ideológico o valorativo de la teoría. De los tipos de principia iuris, los principia iuris et in iure expresan opciones de valores como la igualdad, el de la representación política y la separación de poderes, los cuales son principios de derecho positivo, explícita e implícitamente dictados por las constituciones de los ordenamientos avanzados, pues, como ha dicho, la referencia empírica de su teoría es el constitucionalismo democrático. Al contrario, los principia iuris tantum, que son los únicos que no pertenecen al derecho sino a la teoría, no son en ningún sentido principios valorativos o ideológicos: se trata de los clásicos principios de unidad, de coherencia y completitud, los cuales se refieren a la estructura formal que los ordenamientos jurídicos de hecho no tienen pero que de derecho deben tener, cualesquiera que sean los principios sustanciales y normativos en ellos incorporados como sus deberes jurídicos.

En suma, mientras los principia iuris et in iure son principios axiológicos, aunque no solo de la teoría sino también del derecho, la teoría se limita a explicar el significado y a extraer las implicaciones necesarias; los principia iuris tantum, en cambio, son principios teóricos externos al derecho positivo, pero no consisten en principios axiológicos, aunque sí en tesis que respecto al derecho positivo, con independencia de sus contenidos, reflejan la estructura normativa interna. Es manifiesto, dice Ferrajoli, que la conjunción de estas dos clases de principios evidencian la divergencia interna del derecho entre su deber ser y su ser efectivo: genera la dimen- 
sión pragmática de la teoría de aquel modelo normativo que es el moderno Estado constitucional de derecho. Pero esta dimensión no es en absoluto el fruto de una arbitraria opción ideológica, sino el necesario reflejo de la específica estructura normativa de la democracia constitucional objeto de la teoría. Hacen parte de tal estructura la incorporación y la positivación en la forma de principia iuris de rango constitucional, de estos principios sustanciales de justicia en los cuales las constituciones explicitan cuáles sean las opciones del autor, los valores propios de la democracia (Vol. I, pp. 36-37).

\section{UN ORDENAMIENTO JURÍDICO POSITIVO NOMODINÁMICO Y MULTI- NIVEL: LA INEVITABLE EXISTENCIA DE DIVERGENCIAS NORMATIVAS}

La teoría sostiene que en un sistema nomodinámico la existencia virtual de antinomias y de lagunas es de hecho inevitable. Esta constatación, sin embargo, parece estar en contraste con la misma teoría que se basa en el principio de plenitud y de no contradicción -los denominados principia iuris tantum-, pero Ferrajoli recalca y aclara, que estos principios (externos al derecho positivo) son normativos y no solamente explicativos, y que, por lo tanto, como de hecho acontece, pueden ser violados por la indebida comisión u omisión de los actos que lesionan o que, por el contrario, garantizan las normas sustanciales. En esto no hay nada extraño: en un sistema nomodinámico las normas, sean primarias o secundarias, y las garantías por ellas establecidas, no son deducibles de otras situaciones o normas correlativas, sino que son producidas por actos normativos. Apunta que se trata de un precio necesario dado que sobre eso se funda todo el edificio del garantismo y del Estado de derecho (Vol. I, p. 694).

Con ello el enfoque teórico abarca la visión sociológico-realista del derecho, necesaria para completar la visión teórica y hacerla más explicativa del objeto de estudio de la teoría, cual es el derecho. Pero junto a ello, la inclusión del estudio de la divergencia normativa dada en ordenamientos jurídicos multinivel como propiamente es el constitucionalismo rígido (como ordenamiento), importa hacer una clara sistematización y tratamiento de las lagunas y antinomias como vicios normativos, estableciendo claramente su concepto y al mismo tiempo la forma jurídica para corregirlos y perfeccionar el ordenamiento positivo, en función de los criterios normativos exigidos por la teoría, por sus principia iuris. Con esto se puede decir que la visión sociológico-realista del derecho se halla comprendida en la teoría del autor italiano, pero además se encuentra en ella misma superada, evitando la denominada falacia del realismo jurídico.

Para la teoría axiomática las antinomias y las lagunas no generan un problema de aplicación cualquiera, sino solo problemas no solucionables 
sin modificación del ordenamiento. Ello restringe, desde luego, el campo de denotación de los dos términos: para que se pueda hablar de "antinomia" o de "laguna", el problema de aplicación por ella generado debe resultar insoluble al intérprete sobre la base del derecho vigente. Debe tratarse, precisamente, de la presencia o de la ausencia indebida de una norma que, si no es removida por intervención normativa, no consiente o permite la aplicación de la norma respecto a la cual la antinomia y la laguna son predicables. Antinomia y laguna se caracterizan en suma porque integran la inobservancia -por incoherencia o por incumplimiento- de la prohibición o de la obligación impuesta por una norma sobre la producción (norma supraordinada), haciéndola inaplicable y requiriendo, para su solución, una modificación del derecho vigente. Este es el sentido estricto de laguna y de antinomia en la teoría de Ferrajoli, dentro de un sistema jurídico nomodinámico y multinivel. Antinomia, dice el autor, es el vicio sustancial producto de la indebida decisión de una norma en oposición con una norma sustancial sobre la producción, cuya aplicación supone la "anulación" de la norma en oposición. Este vicio normativo contrario al principia iuris tantum de no contradicción, principio externo de la teoría del derecho, debe ser resuelto en un sistema constitucional avanzado a través de una expedita jurisdicción constitucional que vela por la eficaz anulación de las normas que se hallen en contraste, en oposición, a las normas constitucionales supraordinadas.

Las lagunas por su parte son definidas como el vicio producto de la indebida omisión de la decisión de una norma requerida por una norma sobre la producción, cuya aplicación supone la introducción de la norma faltante. Distingue el autor dos tipos de lagunas: las primarias y las secundarias. En cualquiera de los dos casos la existencia de lagunas supondrá la denominada inefectividad estructural del ordenamiento que se opone al principio de completitud del derecho (principia iuris tantum), y que requiere ser, entonces, detectado, denunciado y corregido, pues este tipo de inefectividad estructural del ordenamiento por regla general alcanza a los derechos sociales y humanos largamente proclamados o reconocidos -en normas supraordinadas-, pero en las normas subordinadas escasamente garantizados -a nivel primario y secundario-. El remedio jurídico a este vicio normativo en un ordenamiento nomodinámico y multinivel (dentro de un modelo de constitucionalismo rígido de veras), es lo que denomina el autor la asunción de la garantía débil, como el deber del legislador de introducir las garantías primarias y secundarias necesarias y correspondientes a las normas supraordinadas; en último término la introducción de las necesarias y debidas normas de legislación de actuación (Vol. I, pp. 684-701).

Por lo anterior es que Ferrajoli afirma: "Es evidente la importancia que reviste el análisis de las antinomias y las lagunas a los fines de la teoría de la democracia constitucional. Son en efecto estos dos vicios 
los que identifican virtualmente el paradigma del constitucionalismo rígido, caracterizado por la divergencia deóntica entre "deber ser" y "ser" del derecho que se manifiesta sobre todo en las antinomias y en las lagunas sobre el derecho respecto a su modelo constitucional." (Vol. I, p. 691).

Es esta divergencia entre niveles normativos diferentes, entre el más alto y más bajo, en el que el último se configura como factual respecto del primero, y en el que el más bajo ha de quedar sometido al primero tanto en la forma como en la sustancia, caracterizado como la sujeción a la ley (supraordinada) de la misma ley (subordinada). La divergencia entre normas de distinto nivel importa un vicio que refleja una imperfección normativa dada entre el "deber ser" (normativo) y el "ser" (normativoefectivo), o sea, entre la "validez" y la "existencia". Tal divergencia puede ser reconocida y sistematizada sin dar lugar a contradicciones, en cuanto se reconozca que es una violación de los límites o de los vínculos impuestos por las normas superiores a aquellas de nivel inferior. En un sistema nomodinámico de derecho positivo dentro del cual la existencia de cada norma no es deducida de otras normas, sino un hecho empírico independiente de su significado, no siempre los principios lógicos de no contradicción y de completitud son satisfechos. Por eso antinomias y lagunas no solo de hecho existen, sino que no pueden no existir entre normas de diverso nivel a causa de la divergencia que siempre subsiste entre "deber ser" normativo y "ser" efectivo (Vol. I, pp. 26-27).

Se comprende, con lo dicho, la calidad o función performativa que se le atribuye a la teoría del derecho en cuanto no solo tiene un fin puramente descriptivo de la realidad que resulta su objeto de estudio, como es el derecho positivo, en cuanto a sus formas, estructuras y conceptos fundamentales, sino además tiene una calidad de performativo que se traduce en la necesidad de que la teoría exprese, explicite o describa el derecho como un "deber ser" normativo, capaz de influir en el mismo derecho. De ahí mismo la función crítica que le atribuye a la dogmática jurídica que ha de analizar e identificar las imperfecciones normativas del derecho vigente. Para ejemplificar Ferrajoli pone el caso de la relación que se formula entre derechos subjetivos y garantías: señala que se establece que la existencia de derechos subjetivos impone la existencia de obligaciones y de prohibiciones que constituyen sus garantías. Pero esta tesis teórica, evidentemente, es descriptiva del "deber ser" del derecho vigente, en el cual es bien posible que falten las previas disposiciones de las obligaciones y de las prohibiciones correspondientes a los derechos estipulados; en ese caso el "deber ser" equivale a la obligación de introducirlo (Vol. I, p. 35). En esta última afirmación se advierte la potencialidad performativa que Ferrajoli le atribuye a la teoría, y la función de identificación concreta que se le concede a la dogmática jurídica de las específicas imperfecciones normativas del derecho vigente. 


\section{LA TEORÍA DE LOS DERECHOS SUBJETIVOS Y DE LAS GARANTÍAS}

Con seguridad el enfoque teórico de esta cuestión resulta una de las aportaciones de mayor relieve que hace el autor. No se puede entender cabalmente su propuesta general sobre el derecho positivo y el Estado de derecho y la interpretación semántica de democracia sin la precisa concepción de los derechos subjetivos y sus garantías. Sin duda una de las cuestiones fundamentales propuesta en la teoría es aquella que se refiere -dentro de la deóntica del derecho- a su compleja y completa proposición respecto a las modalidades deónticas sobre las cuales se sostiene el concepto de derecho subjetivo y de garantías primarias y secundarias.

Para la construcción teórica respecto de estas cuestiones son fundamentales las definiciones de expectativa positiva y negativa y sus correspondientes términos de obligación y prohibición. Es así que para Ferrajoli una definición rigurosa de derecho subjetivo se construye sobre la base de la expectativa positiva o negativa de un comportamiento, por eso define derecho subjetivo como cualquiera expectativa positiva de prestación o negativa de no lesión (correspondiente a un interés positivo, en el primer caso, y, en el segundo, negativo).

La expectativa como elemento nuclear sobre el cual se construye el derecho subjetivo resulta fundamental para comprender el carácter deóntico del derecho en cuanto referido a modalidades que atienden a comportamientos posibles, y no necesarios. Del mismo modo permite comprender de manera cabal el sentido que se establece entre derecho subjetivo y garantías del mismo, y la efectividad normativa del derecho positivo según los patrones ideales de la teoría. De esa manera resulta que garantía primaria es la obligación de prestación o de prohibición de lesión dispuestos como garantías de un derecho subjetivo. Garantía secundaria, a su vez, es la obligación de anulación o de condena predispuestas como garantías de la anulabilidad de un acto inválido o de la responsabilidad de un acto ilícito (Vol. I, pp. 143 y ss. en relación con pp. 639-705). Se establece con claridad que la especificidad del derecho, con respecto a los otros sistemas deónticos, reside en el hecho de que este dispone de un conjunto de garantías jurídicas - primarias y secundarias- destinadas a asegurar la efectividad de las expectativas, en último término, de los derechos subjetivos.

Para el autor todo derecho subjetivo es siempre una "situación subjetiva" que se resuelve en una expectativa de prestación o no lesión, por lo tanto, en una situación pasiva, lo cual es bastante claro en los derechospretensión o en los derechos-inmunidad, pero también aquello es predicable de los denominados derechos-potestad (potestativos) y de los derechos-facultad (facultativos). Afirma que tanto la categoría de los derechos potestad como facultad, para ser verdaderamente considerados derechos subjetivos deben suponer no solo la facultad sino necesariamente además una expectativa. Por eso sostiene que el concepto que aglutina y caracte- 
riza a los derechos subjetivos es el de expectativa. Con ello no se niega la existencia de derechos subjetivos que supongan facultas agendi-facultad o potestad-, pero esa facultad o potestad solo da lugar a un derecho subjetivo en caso que ello importe un interés jurídicamente tutelado a través del deber impuesto a otro u otros de respetarlo. Solo de esta manera se puede entender que un derecho subjetivo que sea facultas agendi constituya en realidad un "permiso fuerte" y, por lo tanto, un derecho subjetivo. De lo contrario nos hallamos ante un simple "permiso débil", o sea, ante una mera facultad extrajurídica siempre posible de legítimas limitaciones o negaciones. Es decir, lo que constituye a una facultad en derecho subjetivo es el establecimiento de una expectativa como forma de tutela jurídica de un interés de un sujeto.

Resulta esencial para la comprensión de la teoría del profesor italiano referirse, junto al concepto de derecho subjetivo y las garantías primarias y secundarias, a la relación que establece entre derecho subjetivo como expectativa y la garantía primaria como obligación de prestación o prohibición de lesión, pues aquello le permitirá explicar en buena medida una aportación esencial a la concepción de derecho subjetivo y su efectividad, lo cual se relaciona con la especial agudeza del autor para demostrar que aquello es fundamental para entender la inevitable estructura nomodinámica del derecho positivo, la cual además se hace más compleja y potencia en un derecho con más de un nivel normativo, como acontece en los Estados constitucionales avanzados.

En Principia iuris (como en obras precedentes) el autor, al distinguir netamente las garantías primarias del mismo derecho subjetivo, como situación jurídica activa no comprendida en el derecho sino autónoma a él, opera una separación de aquello que se suele considerar que se halla naturalmente incluido en el mismo derecho y que justamente lo califica de tal. Pero precisamente aquí está la importante aportación de la teoría, pues esa distinción de la expectativa, propia del derecho, de la garantía misma como obligación o prohibición, separado de este, permite concebir y comprender la estructura nomodinámica del derecho positivo, que se haIla en permanente movimiento hacia la integridad, plenitud y coherencia, dentro de un esquema jurídico multinivel propio del constitucionalismo rígido que impone límites y contenidos a la ley (al legislador); se entiende además la necesidad de lo que se denomina la legislación de actuación para dar efectividad a los derechos fundamentales constitucionalmente consagrados. En último término se comprende así el concepto de laguna (ausencia de garantía) y de antinomia de la ley puesta bajo la norma constitucional supraordinada.

Dice Ferrajoli: "La primera garantía de un derecho reside en suma en el hecho que alguno tenga el deber a él correspondiente; la segunda en el hecho que alguno tenga el deber de dar actuación a la responsabilidad o a la anulación consiguiente a sus violaciones. La estructura garantista del 
ordenamiento deriva de la caracterización de éste como una red compleja de relaciones jurídicas sean singulares o universales, sean horizontales o verticales."

Y añade: “(...) Las garantías primarias, aunque lógicamente implicadas por las expectativas que forman el contenido de los derechos, pueden de hecho faltar, a causa de la estructura nomodinámica de los ordenamientos positivos (...) y, por lo tanto, por su posible incompletitud y contradictoriedad. Es cuanto acontece con un derecho subjetivo que viene establecido sin que sea determinado el (sujeto que tiene) deber correspondiente, y sin por lo tanto que sea concretamente identificada la relación jurídica por esta razón postergada. Así, por ejemplo, muchos derechos sociales, como el derecho al trabajo previsto por el art. 4 de la Constitución italiana, que no comprendieren obligaciones taxativas sobre un sujeto u órganos explícita y exactamente determinados. También, en general, todos los derechos negativos: si faltasen, para la garantía del derecho a la vida o del derecho a la propiedad, normas penales contra el homicidio o contra el hurto, estos actos, por fuerza del principio de estricta legalidad penal, no serían punibles como delitos; hasta la obligación del resarcimiento del daño por efecto de la lesión de un derecho subjetivo tiene el fundamento en una norma, como el art. 2043 del Código civil italiano (...) Incluso si fundamentales y sancionados constitucionalmente, los derechos reclaman siempre una legislación de actuación, es decir, el deber ser jurídico de las garantías primarias representadas por los deberes correspondientes, pena sus indebidas descalificaciones como vacías declamaciones normativas." (Vol. I, pp. 673-674).

El autor añade que las garantías primarias y las conexas relaciones jurídicas (casi) pueden parecer una obviedad cuando se trata de derechos individuales patrimoniales estructurados como derechos reales erga omnes o derechos personales erga singulum, en cuyos casos se da por descontada la existencia e implicación por el mismo derecho de las debidas prohibiciones o prestaciones a cargo de otros como garantías primarias del derecho respectivo. Pero en donde la teoría muestra toda su potencialidad es en el análisis de lo que sucede en el ámbito de los derechos sociales, en el cual ni en el plano teórico ni en el práctico resulta obvia la existencia y plenitud del sistema de garantías primarias (y secundarias), sino bien al contrario, en donde de ordinario los derechos sociales fundamentales suelen estar ampliamente proclamados a nivel constitucional y muy débilmente tutelados por las correspondientes garantías (Vol. I, p. 674).

Para Ferrajoli, sin embargo, la estructura del Estado constitucional se distingue en su modelo normativo por la tutela de las necesidades vitales de las personas a través de su reconocimiento como derechos fundamentales, y por la consecuente configuración de aquella esfera que llama "esfera pública", como un sistema de prohibiciones de lesiones y de obligaciones de prestación, es decir, de límites y vínculos dispuestos como garantías (Vol. I, p. 674). 
Por eso sostiene que de la carencia de garantías primarias, es decir, de las previsiones normativas de los deberes positivos o negativos correspondientes a los derechos constitucionales, además de los sujetos específicamente vinculados, resulta la configuración de indebidas lagunas que deben ser colmadas a través de las idóneas interpretaciones o decisiones (Vol. I, pp. 674-675).

\section{Democracia y DEREChO. IMPORTANCIA DE LA CONCEPCIÓN SOBRE LA DEMOCRACIA FORMAL Y DEMOCRACIA SUSTANCIAL}

La teoría de la democracia desarrollada en el Vol. II, a diferencia de la teoría del derecho, no es formal ni formalizada, sino esencialmente normativa. No es formal ni en el sentido epistemológico ni metateórico del término, ni formalizada desde un punto de vista lógico estructural. La teoría de la democracia es plenamente normativa, pero se sirve del aparato conceptual elaborado por la teoría formal del derecho, de la cual representa una interpretación semántica de tipo axiológica o normativa, en el marco de la experiencia constitucional de las democracias avanzadas (Vol. II, p. 6). Veamos las cuestiones principales para entender la interpretación semántica de democracia.

\section{Democracia formal y democracia sustancial}

En Principia iuris Ferrajoli dice que la democracia formal es concebida como un método de formación de las decisiones públicas, precisamente como el conjunto de reglas que atribuyen al pueblo o, mejor, a la mayoría de sus miembros el poder directo o indirecto de adoptar esas decisiones. La fuente de legitimación del poder, apunta, es la autonomía, o sea la libertad positiva consistente en el gobernarse a sí mismo y no hacer depender de ningún otro distinto de sí mismo la reglamentación de la propia conducta: en los hechos, que las decisiones sean adoptadas directa o indirectamente por sus propios destinatarios o, más precisamente, por la mayoría; con ello esta se convierte en manifestación de la "voluntad" y de la "soberanía popular" (Vol. II, p. 5).

Señala el autor: "Podemos llamar formal o procedimental esta definición de democracia. Ella identifica en efecto la democracia simplemente sobre la base de las formas y de los procedimientos idóneos destinados a garantizar que la decisión producida sea expresión, directa o indirecta, de la voluntad popular. Identifica, en breve, con base en el quién (el pueblo o sus representados) y al cómo (regla de la mayoría) de las decisiones, independientemente de sus contenidos, es decir, de qué cosa viene decidida. Así en un sistema en el cual con la mayoría fuese consentido decidir la reducción de los derechos de la minoría sería, en la medida de este criterio, 
"democrático". La tesis que considero sostener es que esta caracterización solamente formal de la democracia no es suficiente para fundar una definición adecuada, y que necesita, a tal fin, ser integrada con las indicaciones de límites y vínculos de carácter sustancial o de contenido." (Vol. II, p. 5).

Y más adelante añade: "Que la dimensión formal de la democracia cual poder fundado sobre la voluntad popular exprese una connotación necesaria es indudable: se trata (...) de una conditio sine qua non, en ausencia de la cual de "democracia" no se puede hablar. Pero, ees esta la sola condición necesaria? ¿Es suficiente una connotación puramente formal de la democracia para identificar todas las condiciones que se requieren de un sistema político para que pueda ser considerado "democrático" y, más específicamente, como una "democracia constitucional"? Yo pienso que no lo sea." (Vol. II, p. 6).

Seguidamente el autor explica por qué no es suficiente un concepto puramente formal de democracia y por qué es necesario asumir además una visión sustancial de la misma. Para ello ofrece cuatro razones precisas. Son las siguientes.

La primera razón estriba en que la concepción formal de democracia por sí misma no posee valor empírico y consecuentemente capacidad explicativa, ya que esta definición de democracia identifica las connotaciones puramente formales, motivo por el cual no es capaz de explicar las modernas democracias constitucionales. La concepción formal se limitaría a requerir que los poderes públicos sean ejercitados por el pueblo como sea: configura así a la democracia como poder absoluto que no reconoce límites. Ignora por lo tanto el paradigma del Estado de derecho que no admite la existencia de poderes no sujetos a la ley, lo cual importa suponer que no existe "un poder del pueblo" ilimitado. Por eso mismo el autor haciendo esta crítica a la concepción puramente formal de la democracia se pregunta, contrastando a esta con la visión sustancial de democracia, "¿Debemos concluir, con la medida de las definiciones formales de democracia únicamente como "poder del pueblo", que estos sistemas no son democráticos? ¿Que los derechos fundamentales sancionados en constituciones rígidas, siendo un límite heterónomo a la dimensión política de la democracia, son por esto un límite a la democracia tout court, al punto de llegar a convertirse, en cuanto tendencialmente "insaciables", en una negación de ella misma? ¿O no debemos afirmar, al contrario, que justamente en ausencia de tales límites, si quizás podemos hablar de "democracia" en sentido puramente político o formal, no podemos hablar a propósito de aquella como constitutiva de una forma compleja de democracia y hoy generalizada en todos los países como es la "democracia constitucional"?" (Vol. II, pp. 6-7).

La segunda razón es la escasa consistencia técnica del concepto formal de democracia. Afirma el autor que ciertos límites sustanciales son fundamentales para la sobrevivencia de la misma democracia. Y por eso afirma que en ausencia de esos límites relativos a los contenidos de las decisiones 
se arriesga su subsistencia: recuerda que bajo una concepción puramente formal siempre es posible suprimir, con la mayoría, los derechos fundamentales y el mismo sistema democrático.

Sostiene que esta posición se ha intentado rebatir con la tesis de que la concepción formal de democracia supone como precondición o presupuesto el aseguramiento de ciertos derechos que impiden la supresión de la democracia política. Pero si ello es así, es decir, si es verdad que una concepción formal de democracia importa como precondición necesaria la existencia y aseguramiento de determinados derechos fundamentales, resulta que esos presupuestos necesarios sin los cuales no se puede hablar de democracia formal deben ser asumidos en el mismo concepto de democracia formal a definir, con lo cual ya no nos hallamos ante la puridad formal que se proclama, pues se asume en ella parámetros sustanciales (Vol. II, p. 7).

La tercera razón consiste en que la concepción formal ignora el nexo indisoluble de la democracia y de la soberanía popular, y de estas con los denominados derechos primarios o sustanciales, los cuales justamente operan como límite sustancial de la soberanía popular. Así, ha de reconocerse el nexo de la democracia política con los derechos de libertad. No hay democracia política sin derecho a sufragio, pero tampoco sin libertad de pensamiento, de prensa o de reunión. Pero más importante que este nexo obvio de la democracia política con los derechos de libertad es la afirmación de que estos derechos son efectivos en cuanto sean a su vez sostenidos por la garantía de los derechos sociales a prestaciones positivas: el derecho a la educación, el derecho a la salud, entre otros tantos. Considera el autor que sin la satisfacción de estos se desvanecen los derechos políticos y de libertad: se quedan en el papel. No existe la justa participación en la vida pública sin la garantía de lo que Ferrajoli denomina aseguramiento de "mínimos vitales", mínimos que -atendida la realidad ideológica de nuestro país- conviene aclarar no son "mínimos de subsistencia", sino de vida digna que permita la justa participación política y el ejercicio de libertades. Con ello se prueba una relación de sinergia de los derechos políticos y de los individuales negativos con los derechos sociales. Del reconocimiento y aseguramiento de estos resulta de veras una potenciación de los primeros.

La cuarta razón esgrimida en contra de la concepción formal de democracia es que esta conduce a una aporía filosófico-política. Se simplifica la concepción de democracia como una autonomía o autodeterminación popular, es decir, como una suerte de libertad del pueblo de no ser sometido a ningún límite que no sean los deliberados por sí mismo. Pero el "pueblo" es un sujeto colectivo, dice Ferrajoli, que no puede deliberar y decidir sino por la mayoría, y ninguna mayoría puede deliberar sobre aquello que no le pertenece, es decir, sobre la supresión o restricción de normas constitucionales que confieren derechos fundamentales y que pertenecen a todos 
y cada uno de sus destinatarios, y además sus titulares: los derechos fundamentales delimitan la "esfera de lo indecidible" que la soberanía popular no puede sobrepasar arbitrando limitaciones o supresiones de derechos fundamentales. Por eso con lucidez afirma que la ecuación o correspondencia de la autonomía o soberanía del pueblo y el método decisional fundado sobre los principios de mayoría y de representación (desmedidos o ilimitados) tiene "a las espaldas (...) una concepción comunitaria, organicista y holística de la democracia basada sobre una larga serie de asunciones ideológicas: la idea que el pueblo sea un "cuerpo político", una suerte de organismo, un macrosujeto, dotado de propia voluntad homogénea; que los principios de la representación y de la mayoría, antes que simples convenciones más que toda otra idónea a determinar los sujetos mayormente "representativos", sean realmente las formas a través de las cuales se expresa la voluntad general del pueblo cual sujeto unitario y orgánico (...). Si por el contrario abandonamos esta metáfora, es fácil reconocer, con John Stuart Mill, que expresiones como 'autogobierno' y 'poder del pueblo sobre sí mismo' no designan en absoluto "el verdadero estado de las cosas. El 'pueblo' que ejercita el poder no coincide siempre con aquellos sobre los cuales este último viene ejercitado; y el 'autogobierno' de que se habla no es el gobierno de cada uno sobre sí mismo, sino de todos los otros sobre cada uno"." (Vol. II, pp. 8-9).

Por eso Ferrajoli cree que la soberanía popular ha de ser entendida en realidad en dos sentidos bien concretos: uno negativo y otro positivo. Como garantía negativa que "la soberanía pertenece al pueblo" y solamente al pueblo; que reside en el pueblo y solamente en el pueblo (...) significa que el principio de la soberanía popular equivale a una garantía negati$v a$, es decir, a la prohibición para cualquiera de apropiarse o de usurparla: significa que la soberanía, perteneciendo al pueblo entero, no pertenece a ningún otro y ninguna persona singular o grupo de personas -monarca o Parlamento, presidente electo del pueblo o asamblea representativa- puede "apoderarse" de ella (...) para ejercitarla en contra de algunos de sus miembros." (Vol. II, p. 10).

Y respecto al significado positivo de soberanía popular apunta Ferrajoli: "He ya dicho que todos los derechos fundamentales dan formas y contenidos "a la voluntad popular", la cual, en cuanto suma de las voluntades de cada uno de sus miembros, no puede manifestarse auténticamente si no puede expresarse libremente, y no puede expresarse libremente sin ser protegida por las garantías no solo de los derechos políticos y de libertad sino también de los derechos sociales. La fórmula "la soberanía pertenece al pueblo" por lo tanto quiere decir, en este segundo sentido, que ella pertenece al conjunto de sus ciudadanos, es decir, a todas las personas de que se compone el pueblo: pertenece, en breve, a todos y cada uno de los ciudadanos, en cuanto equivale a la suma de aquellos poderes y contrapoderes de todos -los derechos políticos, los derechos civiles, los derechos 
sociales- que son los derechos fundamentales constitucionalmente establecidos. Estos derechos, consecuentemente, no son solamente los límites a su concreto ejercicio, sino son además la sustancia democrática, dado que se refieren al pueblo en un sentido bastante más concreto y lleno de la misma representación política, es decir, se refieren a todos y cada uno de los miembros de carne y hueso." (Vol. II, pp. 10-11).

Considera el autor, con coherencia, que la democracia constitucional tiene un correlato con esta forma de entender la soberanía popular en una dimensión sustancial y por eso apunta: "La democracia constitucional se configura así como un paradigma complejo que incluye (...) junto a la dimensión política o formal, también una dimensión que he llamado sustancial, dado que se refiere a la sustancia de las decisiones, es decir, a los límites (lo que está prohibido) y a los vínculos (lo que es obligatorio) impuesto a los poderes representativos como otros tantos principios axiológicos de la democracia." (Vol. II, p. 12).

Como se ve en la teoría de la democracia de Ferrajoli no se pretende desconocer su dimensión formal, pero junto con ello se reclama la necesidad del aspecto sustancial que recoja la forma avanzada de democracia constitucional que no se satisface con la sola dimensión procedimental, sino que además demanda el reconocimiento de límites a las decisiones o de contenidos vinculantes para ellas. Por eso afirma con claridad: "Las reglas de aquello que he llamado democracia formal son en efecto dirigidas a disciplinar los medios y las formas de la decisiones, que se reservan al poder y la voluntad de los particulares en la vida privada y al poder y la voluntad de la mayoría en la vida pública, quedando indiferentes a los fines y no preordenando la sustancia. Y consisten por esto en la atribución de los derechos secundarios o instrumentales o de autonomía, individual o colectiva, a través de normas formales que condicionan a su vez el válido ejercicio, la innovación y la transformación jurídica. Las reglas de aquello que he llamado democracia sustancial consisten por el contrario en límites y vínculos a la autodeterminación individual, a tutela de intereses o necesidades vitales de todos. Y consisten por lo tanto en la atribución de los derechos primarios o finales o sustanciales, que aquellos intereses y aquellas necesidades reflejan, a través de normas sustanciales que disciplinan el ejercicio de los poderes de autodeterminación, de otra manera absoluta, en las cuales consisten los derechos secundarios. Las reglas del primer tipo, como se ha visto, son las de reconocimiento de los poderes legítimos y de las decisiones formalmente válidas en un ordenamiento democrático; aquellas del segundo expresan la razón social a la que las unas y las otras están en tal ordenamiento subordinadas. Las unas valen para fundar la dimensión nomodinámica, las otras la dimensión nomostática de la democracia constitucional." (Vol. II, p. 17). 


\section{LAS TESIS SOBRE DEMOCRACIA LIBERAL Y DEMOCRACIA SOCIAL}

Un correlato que establece Ferrajoli con claridad es el de la democracia liberal y democracia social con el de los derechos individuales y los derechos sociales. Para el autor es evidente que la primera garantiza con cierta efectividad solo los derechos individuales, mientras que los derechos sociales son asegurados en la democracia social. Pero más interesante de destacar es la idea que estas formas de democracia no se excluyen o no se hallan en tensión antagónica, sino que entre ellas existe una debida sinergia. Así, no existe plena participación política, libertad política o de expresión, sin un efectivo derecho a la educación o a la salud. Se afirma la relación o mutua implicación que se establece entre los dos aspectos de la democracia, el liberal y el social.

Considera que las dimensiones liberales de la democracia son cuatro. Primero, el principio de laicidad del derecho como consecuencia de su separación de la moral y la religión, constituyéndose en instrumento de garantía de las personas ante injustas ofensas (principio de ofensividad de los actos ilícitos o prohibidos).

En segundo lugar se caracteriza por la consagración de las denominadas "libertades de" y junto con ello, y en tercer lugar, por el establecimiento de las denominadas "libertades para". Estas categorías de derechos propios de la democracia liberal se conciben de una parte como puras inmunidades frente a posibles ofensas de otros en el caso de las "libertades de", y en el caso de las "libertades para" no solo como inmunidades sino además cual facultades para actuar. Pues bien, esta concepción fundamental y propia del Estado de derecho liberal importa además la existencia de una relación coherente entre ambos tipos - de inmunidades y facultades de actuación- en donde las primeras limitan a las segundas, pero siempre sobre la base del principio de ofensividad.

El principio de ofensividad opera en dos sentidos: uno positivo y otro negativo. Primero, solo se pueden limitar derechos y libertades para el caso que efectivamente supongan una lesión a alguna inmunidad o libertad negativa consagrada en el derecho positivo. Y, segundo, significa que no se puede limitar un derecho si su ejercicio no importa una lesión a una inmunidad o libertad negativa. Este segundo aspecto se vincula de manera inmediata y directa con el carácter laico del derecho, ya que el principio de ofensividad en su aspecto negativo impide la limitación de derechos por otras razones que se separen de la ofensividad como pueden ser las morales o religiosas o cualquier otro criterio sustancialista.

En cuarto lugar, Ferrajoli pone en evidencia aquello que denomina las garantías primarias y secundarias de los dos tipos de libertad establecidos de conformidad al principio de ofensividad.

A la base de la concepción liberal de democracia está la idea fundamental del pacto basado en el deber del Estado de respetar y proteger el derecho a la vida como la primera razón de ser del Estado y del derecho. 
Las libertades en general consideradas como inmunidades son requeridas por el principio de ofensividad y por la función garantista del derecho. Las "libertades para", que no solo son inmunidades sino también facultades, son la libertad de pensamiento, la libertad de reunión, la de libertad de asociación y la libertad de circulación, se hallan a la base de la concepción de la democracia liberal y operan o son un presupuesto esencial de la democracia política. Sin embargo, para el autor el Estado liberal de derecho, la democracia liberal sin más, responde a un realidad superada y actualmente inexistente. Señala que el corolario del derecho a la vida es el derecho de sobrevivencia, al cual son reducibles de distinta manera gran parte de los derechos sociales a prestaciones positivas por parte de la "esfera pública": como los derechos a la subsistencia, a la previsión, al trabajo, a la salud y a la instrucción (Vol. II, p. 392).

Dice que "En las sociedades modernas, caracterizadas por un alto grado de interdependencia y de desarrollo tecnológico, incluso sobrevivir, no menos que vivir, requiere en efecto garantías jurídicas, y precisamente garantías primarias positivas. También la sobrevivencia, no menos que la defensa de la vida de indebidas agresiones, es siempre menos un fenómeno solamente natural y siempre más un fenómeno artificial y social" (Vol. II, p. 392). Por eso el autor considera que sus garantías deben ser incluidas en la razón social de la democracia constitucional. Apunta que el proceso de civilización tiene una vinculación inmediata con la pérdida progresiva de la autosuficiencia de las personas y con el consecuente crecimiento de la interdependencia social: sobrevivir en sociedad ya no es más un hecho natural y que dependa de la decisión voluntaria del individuo, sino cada vez más un hecho social. Se entiende así, afirma Ferrajoli, que "en la época de Locke, cuando aún existía una relación directa entre vida y naturaleza, la sobrevivencia bien podía ser confiada a la autonomía del individuo: a su trabajo ante todo, a su capacidad de adaptación, a su libertad y responsable iniciativa y en cualquier caso -escribía Locke formulando el más sólido fundamento axiológico del naciente capitalismo- a su voluntad de trabajar y cultivar nuevas tierras 'sin perjuicio de ninguno, puesto que hay tierra suficiente en el mundo para el doble de habitantes' (...). Fue sobre esta base que el primer liberalismo pudo teorizar sobre el nexo entre libertad, trabajo, propiedad y vida, y a cuya 'mutua conservación' está dirigido el contrato social" (Vol. II, p. 392). Y concluye con mucha claridad: "Ciertamente que hoy ya no es más así. 'El hombre civilizado', escribió Tocqueville hace 170 años, 'está infinitamente más expuesto a las vicisitudes del destino que el hombre del pasado'. Bastante más que en el pasado, la entera sobrevivencia del hombre -desde el trabajo hasta la emigración, desde la habitación hasta la alimentación de base- es confiada a su integración social, es decir, a condiciones materiales y circunstancias jurídicas y sociales de vida que van más allá de su libre iniciativa." (Vol. II, pp. 392-393). 
La segunda razón positiva está representada por los enormes progresos tecnológicos y por los crecientes desarrollos de la riqueza y de la producción. En efecto, se atribuye a los progresos de la medicina y de la tecnología la capacidad de mejorar las condiciones de vida y de aumentar la producción de bienes materiales de manera formidable, y a partir de esas circunstancias -se afirma- se ha hecho materialmente posible, bastante más que en el pasado, reducir o eliminar las desigualdades, las desigualdades de oportunidades, y esa posibilidad material convierte su articulación política en un imperativo moral y políticamente debido. Es decir, se impone el deber de eliminar las desigualdades de oportunidad que dependen de múltiples factores, muchos de ellos ajenos al control de las personas, a la autodeterminación del individuo, y que responden más bien a la "lógica del azar" o, parafraseando a Rawls (dice Ferrajoli), a la "lotería natural", así como las desigualdades de dotes naturales de las personas, como son las físicas, las de salud o de fuerza, o directamente las desigualdades sociales debidas al origen sociocultural o debidas al lugar de nacimiento, entre otras (Vol. II, p. 393).

Son estas las razones fundamentales por las cuales Ferrajoli estima que el Estado social de derecho, la democracia social importan una ampliación de la tutela ofrecida por el Estado liberal y su democracia. Una necesidad demandada por el carácter social y artificial de la sobrevivencia del individuo. Es en ese esquema que "La democracia social representa una ampliación del paradigma del Estado de derecho." (Vol. II, p. 394).

Tras esta explicación sobre la democracia liberal y democracia social, conviene resaltar que lo más sobresaliente de la teoría comentada está en el desarrollo exhaustivo y claro de los problemas de efectividad que presentan los derechos sociales que caracterizan a la democracia social: el aseguramiento o tutela de ellos a través de las correspondientes garantías primarias y secundarias. Desde esta perspectiva los problemas de las garantías sociales primarias son sobre todo de carácter económico y político. El problema económico es que, como su nombre indica, ellos tienen un costo económico más que las garantías de los derechos de libertad. El segundo problema estriba en que la satisfacción de los derechos sociales importa, frecuentemente, la mediación burocrática que es fuente de discrecionalidad y de corrupción, de inequidad y de inefectividad.

\section{Respecto al problema (costo) económico de los derechos sociales}

El autor sostiene que resulta tal argumento una manera desenfocada de análisis. Reconoce que los derechos sociales tienen un costo para el Estado, lo cual parece una realidad innegable, pero señala al mismo tiempo que los demás derechos, incluidos aquellos típicos de los Estados liberales también lo tienen: lo tienen las libertades y lo tienen los derechos políticos. Asumiendo entonces esta constatación, sin embargo, precisa que 
el criterio del costo económico de los derechos no dice nada acerca de la necesidad de su satisfacción: el crecimiento económico y la producción de la riqueza siendo necesarios no son fines en sí mismo ni menos parámetros de legitimidad de los derechos. Los fines de los derechos, precisa, es la actuación de la razón social de la democracia constitucional (avanzada, que no es el caso de la chilena, desde luego) establecida por su estatuto constitucional, como son la garantía de la paz y de los derechos vitales -políticos, civiles, de libertad y sociales- constitucionalmente establecidos (Vol. II, p. 68).

Por otra parte añade que en realidad si bien se mira el problema del costo de los derechos sociales se advierte que entre la satisfacción de estos y el desarrollo económico y bienestar la existencia de una sinergia evidente, lo cual se halla ampliamente demostrado a lo largo de la historia. Por eso refuta de manera bastante radical la ideología liberal que sostiene que el desarrollo económico viene asociado al solo ejercicio de la autonomía empresarial, como si únicamente el mercado fuera factor de la producción de riqueza: el libre intercambio de productos y servicios y el aprovechamiento del trabajo solo permite la acumulación para el desarrollo de las estructuras y de las infraestructuras productivas, pero resulta una visión marcadamente reduccionista de las causas del desarrollo, más todavía que aquella que mide el crecimiento por el solo aumento del PIB. Utiliza en esta línea de argumentación la experiencia de desarrollo histórico que demuestra que los más importantes períodos de desarrollo económico y bienestar han venido acompañados de importantes procesos de ampliación en la satisfacción de derechos sociales. De eso son ejemplos los casos de Japón y China en distintos momentos, cuyos impresionantes procesos de desarrollo, se ha probado, estuvieron vinculados a procesos de ampliación en la satisfacción de los denominados mínimos vitales. De esta manera el gasto social, el costo de la satisfacción de derechos sociales debe ser considerada una inversión pública: de esa manera la satisfacción de los derechos sociales no solo resulta un fin en sí mismo constitucionalmente exigida en las democracias constitucionales avanzadas, sino además un medio de desarrollo económico, la mejor política económica, aquella más eficaz para promover el desarrollo (Vol. II, pp. 68-71).

\section{El segundo problema respecto a la eficacia de los derechos sociales: el problema político}

Frente a un esquema paternalista, discrecional y asistencial de Estado que concede, potestativamente, prestaciones positivas decididas discrecionalmente por el gobierno, de conformidad a criterios de oportunidad política, y determinada la amplitud de su titularidad (o cobertura) de la misma manera, el autor considera que la construcción de un auténtico Estado social de derecho y democracia social importa que la base o funda- 
mento de legitimidad de los derechos sociales ya no se encuentre en la representatividad política o en el principio de mayoría, sino en el principio de legalidad. Con ello se asegura el carácter vinculante de los mismos y la naturaleza universal de sus garantías. Se asegura además el carácter cognitivo o recognitivo de la justiciabilidad (de la garantía secundaria) de los derechos sociales.

En segundo lugar, la teoría de Ferrajoli señala que junto al principio de legalidad (que asegura el carácter vinculante, universal, no discrecional y la función recognitiva de la garantía secundaria: justiciabilidad) debe asegurarse la separación orgánica y funcional de la satisfacción de los mismos de la función de gobierno que se encuentra dentro de la esfera de la decisión y control político, con el propósito de hacer efectiva la máxima reducción de la discrecionalidad político-administrativa de las prestaciones destinadas a garantizar derechos sociales. Con ello se reduce la mediación burocrática que afecta o lesiona el carácter vinculante y universal tal como hemos hablado. Se propone entonces la necesaria creación de una red orgánica adecuada para la correcta satisfacción de los derechos sociales, sometida en el cumplimiento de su función al principio de legalidad, ajena a la decisión política propia del gobierno-ejecutivo. Por eso dice el autor que "El verdadero problema de los derechos sociales -seguramente el más grave y difícil- es que sus garantías primarias positivas requieren en todo caso una legislación de actuación, es decir, la introducción, justamente, de las instituciones y de las funciones adecuadas para su satisfacción" (Vol. II, p. 400).

Para concluir este comentario solo debemos señalar que se ha querido hacer una síntesis explicativa de las ideas y conceptos más relevantes contenidos en la extraordinaria obra que es Principia iuris, pero debo advertir que he decidido omitir infinidad de aspectos teóricos de la propuesta integral de Ferrajoli que merecen, tal vez, la misma atención por la sustancialidad que poseen dentro de la teoría como es, por ejemplo, la completa exposición que se hace, en el Capítulo XVI, sobre la democracia y la globalización, vinculando la democracia como forma supranacional de gobierno y la repercusión y vinculación que ello tiene con una forma de "constitucionalismo global".

Carlos del Río Ferrettit ${ }^{*}$ 